\title{
Clinical Impact of Liraglutide as a Treatment of Obesity
}

This article was published in the following Dove Press journal:

Clinical Pharmacology: Advances and Applications

\author{
Heshma Alruwaili (D' \\ Babak Dehestani \\ Carel W le Roux ${ }^{1,2}$ \\ 'Diabetes Complications Research \\ Centre, Conway Institute, University \\ College Dublin, Dublin, Ireland; \\ ${ }^{2}$ Diabetes Research Group, School of \\ Biomedical Sciences, Ulster University, \\ Belfast, UK
}

\begin{abstract}
Obesity is defined as a chronic, complex, relapsing disease characterized by excessive adipose tissue. Obesity impacts an individual's health by increasing complications such as prediabetes, type 2 diabetes mellitus (T2DM), hypertension, dyslipidemia, metabolic syndrome, cardiovascular disease, nonalcoholic fatty liver disease (NAFLD), cancers (eg endometrial), and obstructive sleep apnea (OSA). With the increase of obesity prevalence and its negative influences on individuals' quality of life, there is a great need for therapy with a purpose to produce sustainable weight loss of more than $10 \%$ in order to improve or even reverse the progress of obesity related complications. The GLP-1 analogue, liraglutide reduce food consumption, promote weight reduction and improve metabolic functions. The primary mechanism of GLP-1 effect on food intake, metabolism, and weight reduction is mainly due to its actions on peripheral (vagal) and central pathways and activation of hindbrain and hypothalamus. The average weight reduction induced by liraglutide was significant and the weight loss was maintained as long as the patients on therapy. Liraglutide has advantages on weight loss maintenance and promoting cardiovascular disease (CVD) risk reduction, by decreasing systolic blood pressure and glycemic index. In this review, we aim to explain the mechanism of action of Liraglutide, its pharmacokinetic properties, its clinical impact on obesity and its safety and tolerability.
\end{abstract}

Keywords: liraglutide, obesity, weight loss, GLP-1 analogue
Correspondence: Heshma Alruwaili Email alruwaily85@hotmail.com
Obesity is defined as a chronic, complex and relapsing disease, characterised by excessive adipose tissue resulting in ill health. Obesity associated with adverse metabolic, biomechanical, and psychosocial health consequences. ${ }^{1,2}$ Previously obesity was merely considered a behavioral disorder or lifestyle choice caused by increased calorie intake or reduced physical activity. However, current evidence which considers obesity as a disease suggests that obesity is mainly caused by a disruption of homeostatic control of body weight and a failure to prevent excessive fat accumulation. ${ }^{3}$ Obesity is associated with impaired metabolic pathways along with disordered signaling of appetite, hence individuals with obesity experience symptoms that manifest as an increase in hunger and lack of fullness. $^{4}$

Obesity is a multifactorial disease caused by many contributing factors such as biological, genetic, epigenetic, environmental, neurological, and hormonal. These cause an imbalance between caloric intake and energy expenditure. ${ }^{5,6}$ Obesity impacts an individual's health by increasing complications such as prediabetes and type 2 diabetes mellitus (T2DM), hypertension, dyslipidemia, metabolic syndrome, cardiovascular disease, polycystic ovary syndrome (PCOS), nonalcoholic 
fatty liver disease (NAFLD), cancers (eg endometrial cancer), obstructive sleep apnea (OSA), depression, and osteoarthritis. $^{7-11}$

The repetitive episodes of hunger and fullness are mainly controlled by the central nervous system (CNS). The subcortical areas of the brain such as the brainstem and the hypothalamus are the regions of the CNS responsible for energy homeostasis control, which receive peripheral neural and hormonal signals about nutritional state and adiposity. ${ }^{12}$ The arcuate nucleus (ARC) of the hypothalamus has an important role in the regulation of food consumption and energy homeostasis. ${ }^{13}$ There are two different groups of neurons responsible for appetite regulation in the ARC including the proopiomelanocortin (POMC) appetite-inhibiting/Anorexigenic neurons and the neuropeptide Y (NPY) and agouti-related peptide (AgRP) appetite stimulating/orexigenic co-expressing neurons. ${ }^{14}$ Neural and hormonal signals from the periphery result in changes in the relative activity of these two neuronal subgroups and the release of their respective neuropeptides, subsequently influencing food intake and energy expenditure. $^{15}$

Satiety gut hormones are part of the mechanisms by which the periphery inform the centre about energy balance. One of these satiety gut hormones is glucagon-like peptide 1 (GLP-1), which is secreted from the intestinal mucosa of endocrine $L$ cells in the ileum and colon in response to the food intake and presence of nutrients in the intestinal lumen. Once secreted, the half-life of GLP-1 is only a few minutes due to the rapid breakdown by dipeptidyl peptidase-4 (DPP4). ${ }^{16,17}$ Liraglutide, a GLP-1 analogue, is $97 \%$ homologous to the amino acid sequence of endogenous human GLP-1. Liraglutide has a prolonged half-life of 13 hours because it is resistant to DPP-4 degradation. As a consequence, the suitable dose of liraglutide is once-daily. ${ }^{18}$ The receptors of liraglutide are Similar to endogenous GLP-1, liraglutide binds to and activates the GLP-1 receptor in the peripheral and central nervous system, pancreas, intestine, stomach, kidney, and heart. ${ }^{19}$

\section{Mechanism of Action of Liraglutide on Food Intake Reduction}

In addition to the action on pancreatic and gastric receptors, liraglutide acts on the brain's subcortical areas to produce satiety. ${ }^{20,21}$ GLP-1 Receptors (GLP-
$1 \mathrm{Rs})$ are located on the nodose ganglion of abdominal vagal afferent nerve fibers that terminate in the nucleus tractus solitarius (NTS) in the brainstem. Information from the NTS is transmitted to the hypothalamus and other forebrain regions through ascending second-order neurons. The results of rodent studies revealed that central or peripheral administration of GLP-1R agonists decreases short-term food and water intake and causes body weight reduction. ${ }^{22}$ In rats and primates' studies, GLP-1 receptors have been identified in different areas of the brain, involving the hypothalamic nuclei and brainstem. ${ }^{23}$ The highest level of GLP-1 receptor immunoreactivity is found in select brainstem and hypothalamic regions that control feeding, including hypothalamic nuclei of the paraventricular and arcuate nucleus as well as the area postrema, NTS, and vagus dorsal motor nucleus. ${ }^{24}$ GLP-1 receptors are located in different areas of the brain including the parietal cortex, hypothalamus and medulla. Liraglutide activates these receptors in the brain that relate to highly desirable food signals in humans. ${ }^{25}$ Box 1 summarises the Glucagon-like peptide receptors in the brain: controlling food intake and body weight.

GLP-1 also increases fullness. Peripheral GLP-1 can reach the central nervous system at the level of the circumventricular organs. ${ }^{26}$ A $100-\mathrm{mm}$ visual ana$\log$ scale assessed satiety in participants receiving GLP-1 analogues. Participants had to indicate their opinion using the following statements: "I cannot eat another bite" (satiety), "I am totally full" (fullness), and "I have never been more hungry" (hunger). Compared to the control group who received a saline infusion, the participants who received GLP-1 were more likely to report satiety and fullness and less likely to report hunger. ${ }^{27}$ The subjects with obesity who administered GLP-1 had a prolonged period of postprandial satiety and a slower rate of gastric emptying than subjects who received saline infusions. ${ }^{28}$ GLP-1 also induced satiety in rodents, while the use of a GLP1 receptor antagonist, exendin 9-39, caused these effects to be dissipated, supporting the role of the GLP-1 receptor in food intake. GLP-1 receptor's mRNA has been identified in several areas of interest in appetitive behaviour and food intake, namely the paraventricular, arcuate nuclei, and supraoptic nuclei. ${ }^{29}$ 
Box I Glucagon-Like Peptide Receptors in the Brain: Controlling Food Intake and Body Weight

\begin{tabular}{l} 
The nodose ganglion of abdominal vagal afferent nerve fibers \\
\hline The area postrema \\
\hline Nucleus tractus solitarius (NTS) \\
Hypothalamic nuclei of the paraventricular and arcuate nucleus which \\
include: \\
Pro-opiomelanocortin (POMC) appetite-inhibiting/Anorexigenic \\
neurons. \\
Neuropeptide Y (NPY) and agouti-related peptide (AgRP) appetite \\
stimulating/orexigenic co-expressing neurons.
\end{tabular}

The mechanism of action of GLP-1 on food intake reduction is mainly mediated through both peripheral and central nervous system (CNS) pathways. Liraglutide directly stimulates POMC neurons and inhibits neuropeptide-Y (NPY) and Agouti-related peptide (AgRP) neurons in the arcuate nucleus resulting in a reduction in hunger and increases in fullness. ${ }^{30}$ These actions can also be associated with effects on other brain areas such as the mesolimbic system resulting in less food-induced reward signals and hence decreased appetitive or food-seeking behaviour. ${ }^{31}$ GLP-1 receptor agonists systemic administration slows gastric emptying, decreases food intake, and reduces body weight. ${ }^{22,32,33}$ Of note, in terms of desirable amount of weight loss, the Look AHEAD study showed that $5 \%$ weight loss did not reduce cardiovascular events, but that $10 \%$ weight loss was sufficient to achieve reduction in events. ${ }^{34}$

In rodent's experimental follow-up study, fluorescently labeled liraglutide was used to assess if peripherally administered liraglutide could reach brain regions that might be involved in its effects on food intake and body weight. Labeled liraglutide appeared exclusively in several brain regions that play an important role in the regulation of feeding including, the paraventricular nucleus of the hypothalamus and central nucleus of the amygdala. Surprisingly, the fluorescently labeled liraglutide was not detected in NTS, a brain region involved in GLP-1's effects on food intake. This would suggest that liraglutide activation of NTS GLP-1Rs may be through an indirect rather than a direct pathway. ${ }^{35,36}$

\section{Pharmacokinetic Properties}

The liraglutide structure includes Arg 34 replacing Lys 34 at the N-terminal and a fatty acid chain is added to Lys
$26 .{ }^{37}$ In contrast to natural GLP-1, this change allows for $99 \%$ albumin binding, allowing liraglutide to escape glomerular filtration and extend its duration of action. Both self-association and decreased renal clearance of the peptide results in an enhanced half-life of approximately 13 hours after subcutaneous injection, compared with the very short half-life of native GLP-1. Liraglutide is detected in the urine and feces as a low level of metabolites, suggesting slow degradation into small peptides, amino acids, and fatty acid fragments eliminated through the liver or the kidney. ${ }^{38}$ Pharmacokinetics of liraglutide have been studied in several sub-populations representing a variety of demographic variables. Overall, the only two factors impacting pharmacokinetics are body weight and sex. Two Phase III studies, one conducted in US and another in Asia showed a decrease in mean exposure (based on AUC values) as body weight increased. ${ }^{39,40}$ However, there was a significant overlap in exposure values between individuals in subgroups of low and high body weight, hence dose adjustment according to body weight (or body mass index) is not required. In the two population pharmacokinetic analyses of the subjects, mean liraglutide exposure was greater for female than male individuals after correction for differences in body weight. As there was generally a large overlap in exposure values among individual male and female subjects, dose adjustment according to sex is not considered to be meaningful. ${ }^{41,42}$

Liraglutide exposure is not related to age, the AUC (adjusted for body weight) was equivalent in young age groups (18-45 years, both included) and older age groups (age 65 years) in a single-dose study in healthy subjects. ${ }^{43}$ Among children with type 2 diabetes, liraglutide exposure in age group 10-17 years is similar to that observed in adults. $^{44}$

In the LEAD-6 trial, exenatide was directly compared with liraglutide to determine the efficacy and safety of each agent. Liraglutide has minimal impact on renal function due to its chemical structure. ${ }^{45}$ Liraglutide was also investigated in subjects with mild, moderate, severe, or no hepatic impairment. 40 Subjects received $0.75 \mathrm{mg}$ of liraglutide as a single dose, and were evaluated after a 72-hours to determine if hepatic impairment influenced liraglutide's kinetic and safety profile. The conclusion was that no hepatic or renal dosing adjustments are necessary with liraglutide. ${ }^{46}$

In a pooled analysis of Liraglutide Effect and Action in Diabetes (LEAD) clinical trials, low-level antibodies to liraglutide were detected (8.7 of patients taking $1.2 \mathrm{mg}$ 
and $8.3 \%$ of patients taking $1.8 \mathrm{mg}$ ). Anti-liraglutide antibody presence did not attenuate the glycemic response to liraglutide, and no patients with anti-liraglutide antibodies had serious or systemic immunologic adverse events. ${ }^{47}$

In vitro studies, liraglutide has shown a very low potential for pharmacokinetic drug-drug interactions related to cytochrome P450 (CYP). Although the slight prolongation in gastric emptying with liraglutide may affect the extent of absorption of co-administered drugs, no clinically relevant interactions were observed between steady-state liraglutide $(1.8 \mathrm{mg} /$ day $)$ and single doses of insulin detemir, atorvastatin, griseofulvin, paracetamol, digoxin, lisinopril or oral contraceptives. ${ }^{12,13}$ When warfarin is administered to patients receiving liraglutide, more frequent monitoring of the international normalized ratio is recommended, because interaction between the two drugs has not yet been evaluated. ${ }^{48}$

\section{Clinical Impact of Liraglutide as Treatment of Obesity}

Several studies were conducted in a large population of subjects with diabetes to evaluate liraglutide as a treatment for diabetes and found that it improved glycemic control and produced significant body weight loss. ${ }^{49}$ Liraglutide was firstly approved for the treatment of diabetes, and several studies were then conducted to evaluate it as a therapy for obesity. The 56 weeks SCALE Diabetes trial which included 846 participants with body mass index of 27 or greater demonstrated that both the 3.0 and $1.8 \mathrm{mg}$ subcutaneous doses of liraglutide performed better than placebo, with percentage body weight reduction of $6 \%, 5 \%$ and $2 \%$, respectively. A total of $50 \%$ of individuals on $3.0 \mathrm{mg}$ liraglutide showed $\geq 5.0 \%$ of body weight reduction, compared with $36 \%$ in individuals on the lower dose and $14 \%$ on the placebo. A total of $23 \%, 14 \%$ and $4 \%$ of participants lost $>10 \%$ body weight in the three arms, respectively. ${ }^{50}$ The SCALE Obesity and Prediabetes trial involving 3731 patients showed that the once-daily subcutaneous dose of $3.0 \mathrm{mg}$ liraglutide, when used as an adjunct to a reduced-calorie diet and increased physical activity, was associated with significant weight reduction in adult with overweight or obesity who did not have diabetes. The response to liraglutide was similar in subjects with prediabetes versus one without prediabetes, and was similar across baseline Body mass index (BMI) categories. The mean weight change with liraglutide was $8 \mathrm{~kg}$ and was generally maintained for 1 year, as long as the patients continued treatment and weight regain usually follows after treatment discontinuation. ${ }^{51}$

The SCALE Sleep Apnoea study demonstrated a reduction in weight and improvement in Apnea Hypopnea Index (AHI) in the liraglutide group compared to placebo. ${ }^{52}$

Weight loss of more than $10 \%$ in individuals with obesity starts to improve other obesity-related-complications, such as reducing cardiovascular disease risk and nonalcoholic steatohepatitis. ${ }^{53}$ The treatment regimens to help patients achieve at least 7\%weight loss could help patients achieve remission of T2DM and prevent patients with prediabetes from progressing further. ${ }^{54}$ The effect of liraglutide in the risk reduction of T2DM in patients with prediabetes was reported in the SCALE prediabetes trial. At week 160 of the study, $2 \%$ of subjects treated with subcutaneous liraglutide $3.0 \mathrm{mg}$ developed T2DM compared to $6 \%$ of subjects in the placebo arm. Liraglutide showed a $80 \%$ reduction in T2DM risk and the estimated time to onset of T2DM over 160 weeks was 2.7 times longer with the liraglutide group than with the placebo group. ${ }^{53}$ The results of this trial confirmed that liraglutide as a weight loss agent improves various cardio-metabolic parameters and reduces the risk of diabetes in individuals with obesity and prediabetes. ${ }^{51}$

In a large, double blind, multicenter trial, 9340 subjects with type 2 diabetes and high cardiovascular risk were randomised to receive subcutaneous liraglutide $1.8 \mathrm{mg}$ daily or placebo and followed for 3.8 years. Death from cardiovascular causes, nonfatal myocardial infarction, or nonfatal stroke was the study's primary outcome. The primary outcome occurred in fewer subjects in the liraglutide arm (13\%) than in the placebo arm $(15 \%)$. Fewer overall deaths (HR 0.85) as well as cardiovascular deaths (HR 0.78) were reported in the liraglutide group compared with placebo. Importantly, there was no increase in hospitalisation due to heart failure in the liraglutide group. This study used liraglutide dose of $1.8 \mathrm{mg}$ as indicated for T2DM and not the dose of $3.0 \mathrm{mg}$ indicated for the treatment of obesity. ${ }^{55}$

In the SCALE maintenance, trial patients with obesity or overweight who lost $\geq 5 \%$ of their initial weight during a low calorie-diet were randomly assigned to $3.0 \mathrm{mg}$ subcutaneous dose of liraglutide or placebo for 56 weeks. The study results showed that liraglutide compared with placebo, improved weight loss maintenance and induced additional reductions in cardiovascular disease (CVD) risk factors such as a reduction in systolic blood pressure, 
fasting plasma glucose and high-sensitivity C-reactive protein (CRP). ${ }^{56}$ At week 56 of the study, participants in the liraglutide arm experienced an additional weight reduction of $6 \%$ from the run-in period compared with a $0.05 \%$ weight reduction in the placebo arm. The percentage of patients who maintained their weight loss from the run-in period was also higher in the liraglutide arm (81\%) compared with placebo (49\%). ${ }^{56}$ Another study in primary care provides the first evaluation of the effect of intensive behavioral therapy (IBT) delivered alone (with placebo) or in combination with liraglutide $3.0 \mathrm{mg}$. After 1 year, mean weight loss in patients who received liraglutide $3.0 \mathrm{mg}$ plus IBT was $7.5 \%$ compared to a $4 \%$ in those who received IBT alone. ${ }^{57}$

\section{Safety and Tolerability}

Generally, GLP-1 agonists and analogues are well tolerated. The most common side effect is nausea which is more frequent in the early stages of therapy. ${ }^{45,49}$ Other gastrointestinal complaints such as constipation, vomiting, and diarrhoea are less common than nausea. ${ }^{45,49,58}$ While these side effects are not a serious health concern, they may contribute to a patient's decision to discontinue therapy. Other side effects include a small but sustained increase in heart rate $(2-4$ beat $/ \mathrm{min})$, this chronotropic effect of liraglutide may be mediated through the GLP-1 receptor on the sinoatrial node. Despite this, studies have reported that GLP-1 agonists through its beneficial effects on weight loss, improvement in metabolic parameters, and blood pressure reduction has a positive effects on the cardiovascular outcomes. $^{59}$ Accordingly, the LEADER study demonstrated a significant reduction in cardiovascular mortality after liraglutide. ${ }^{55}$

Liraglutide is associated with a low risk of hypoglycaemia. Very few minor or major hypoglycaemic episodes have been reported with liraglutide across the LEAD studies. For example, as a monotherapy, no major hypoglycaemia incidents were reported and only $8 \%$ of patients treated with liraglutide $1.8 \mathrm{mg}$ reported minor hypoglycaemia (plasma glucose $<3.1 \mathrm{mmol} / \mathrm{l}$ ), corresponding to 0.25 episodes/subject-year. In contrast, $24.2 \%$ of glimepiridetreated subjects reported minor hypoglycaemia; the rate was p1.96 episodes/subject-year. ${ }^{60}$ No major hypoglycaemia was reported when liraglutide was used in combination therapy with metformin. ${ }^{61}$ The LEADER study showed that nephropathy events were $22 \%$ lower after liraglutide therapy compared to placebo, but there was no significant difference in retinopathy events. ${ }^{55}$
Few minor and major hypoglycaemic episodes have been reported with liraglutide across the LEAD studies. ${ }^{28}$ For example, as a monotherapy, no major hypoglycaemia incidents were reported and only $8 \%$ of patients treated with liraglutide $1.8 \mathrm{mg}$ reported minor hypoglycaemia (plasma glucose $<3.1 \mathrm{mmol} / \mathrm{l}$ ), corresponding to 0.25 episodes/subject-year. In contrast, $24.2 \%$ of glimepiridetreated subjects reported minor hypoglycaemia; the rate was p1.96 episodes/subject-year $(\mathrm{p}<0.0001) .{ }^{28}$ Only one major hypoglycaemic episode (blood glucose $1 / 43.0 \mathrm{mmol} /$ 1) was reported when liraglutide was used in combination with one OAD.

Additionally, since GLP-1 receptor agonists can cause significant weight loss in a short period, cholecystitis has been reported in 3 in 100 patients. A known serious side effect of gallstone formation is pancreatitis. Generally, weight loss by any modality (diet, medication or surgery) can result in gallstone formation and gallstones are a known aetiological factor for pancreatitis even after bariatric surgery. ${ }^{62}$

However, in a clinical trial involving approximately 4500 patients, only seven cases of pancreatitis were reported which was not more than what can be expected in this population of patients. Reviews of clinical data by the European Medicine Agency (EMA) concluded that treatment with GLP-1 receptor agonists did not increase the risk of developing pancreatitis. ${ }^{63}$ Rodent studies demonstrated proliferation of thyroid C-cells and tumor formation following long-term treatment with GLP-1 agonist compounds, but the potential risk of thyroid cancer has not been shown in non-human primates or humans. ${ }^{64}$ This may be because humans have a lower density of GLP-1 receptors on thyroid C cells. ${ }^{65}$ However, the side effects of prolonged use of higher doses of liraglutide such as those used for weight loss treatment in humans requires long-term surveillance.

In conclusion, the GLP-1 analogue, liraglutide, decreases food intake, promotes weight loss, and improves metabolic function parameters. The primary mechanism of GLP-1 effect on food intake, metabolism and weight reduction is mainly due to its actions on peripheral (vagal) and central pathways and activation of hindbrain and hypothalamus, and those brain regions associated with motivation and reward processes. With the high prevalence of obesity and its negative impacts on the quality of life, there is a need for therapies that will produce sustained double digit weight loss. Bariatric surgery is currently the most effective treatment option for diabetes 
and obesity. Comparing the clinical efficacy of bariatric surgery and liraglutide it is clear that surgery is superior to liraglutide for both body weight reduction and improvements in metabolic parameters. ${ }^{66}$ Despite this, many individuals are not eligible for surgery and would benefit from anti-obesity medications. Liraglutide produces significant weight loss in patients with and without T2DM and has tolerable side effects. Liraglutide is an attractive treatment option, although the GLP-1 class of medication may hold even more promise in the near future.

\section{Disclosure}

Professor Carel W le Roux is advisory board member for Novo Nordisk, GI Dynamics, Boehringer Ingelheim, Herbalife, Keyron, Johnson \& Johnson, and AnaBio. The authors report no other conflicts of interest in this work.

\section{References}

1. O'Rahilly S, Farooqi IS. Human obesity: a heritable neurobehavioral disorder that is highly sensitive to environmental conditions. Diabetes. 2008;57(11):2905-2910. doi:10.2337/db08-0210

2. Pilitsi E. Pharmacotherapy of obesity: available medications and drugs under investigation. Metabolism. 2019;92:170-192. doi:10.1016/j.metabol.2018.10.010

3. Jéquier E, Tappy L. Regulation of body weight in humans. Physiol Rev. 1999;79(2):451-480. doi:10.1152/physrev.1999.79.2.451

4. Blundell J, Lawton C, Hill A. Mechanisms of appetite control and their abnormalities in obese patients. Horm Res Paediatr. 1993;39 (Suppl. 3):72-76. doi:10.1159/000182788

5. Bray GA, Frühbeck G, Ryan DH, Wilding JPH. Management of obesity. Lancet. 2016;387(10031):1947-1956. doi:10.1016/S01406736(16)00271-3

6. Hales CM, Fryar CD, Carroll MD, Freedman DS, Aoki Y, Ogden CL. Differences in obesity prevalence by demographic characteristics and urbanization level among adults in the United States, 2013-2016. JAMA. 2018;319(23):2419-2429. doi:10.1001/jama.2018.7270

7. Narayanaswami V, Dwoskin LP. Obesity: current and potential pharmacotherapeutics and targets. Pharmacol Ther. 2017;170:116-147. doi:10.1016/j.pharmthera.2016.10.015

8. Boutari C, Mantzoros CS. Inflammation: a key player linking obesity with malignancies. Metabolism. 2018;81:A3-A6. doi:10.1016/j. metabol.2017.12.015

9. Huh JH, Kim KJ, Kim SU. Obesity is more closely related with hepatic steatosis and fibrosis measured by transient elastography than metabolic health status. Metabolism. 2017;66:23-31. doi:10.1016/j.metabol.2016.10.003

10. Dhana K, Nano J, Ligthart S. Obesity and life expectancy with and without diabetes in adults aged 55 years and older in the Netherlands: a prospective cohort study. PLoS Med. 2016;13(7):e1002086e1002086. doi:10.1371/journal.pmed.1002086

11. Koren D, Taveras EM. Association of sleep disturbances with obesity, insulin resistance and the metabolic syndrome. Metabolism. 2018;84:67-75. doi:10.1016/j.metabol.2018.04.001

12. Crane J, McGowan B. The GLP-1 Agonist, Liraglutide, as a Pharmacotherapy for Obesity. Vol. 7. London, England: SAGE Publications; 2016:92-107.

13. Chaudhri OB, Salem V, Murphy KG, Bloom SR. Gastrointestinal satiety signals. Annu Rev Physiol. 2008;70(1):239-255. doi:10.1146/ annurev.physiol.70.113006.100506
14. Small CJ, Bloom SR. Gut hormones and the control of appetite. Trends Endocrinol Metab. 2004;15(6):259-263. doi:10.1016/j. tem.2004.06.002

15. Chaudhri OB, Wynne K, Bloom SR. Can gut hormones control appetite and prevent obesity? Diabetes Care. 2008;31(Supplement 2):S284-S289. doi:10.2337/dc08-s269

16. Deacon CF, Johnsen AH, Holst JJ. Degradation of glucagon-like peptide- 1 by human plasma in vitro yields an $\mathrm{N}$-terminally truncated peptide that is a major endogenous metabolite in vivo. $J$ Clin Endocrinol Metab. 1995;80(3):952-957.

17. Holst JJ, Deacon CF. Glucagon-like peptide-1 mediates the therapeutic actions of DPP-IV inhibitors. Diabetologia. 2005;48(4):612-615. doi:10.1007/s00125-005-1705-7

18. Knudsen LB, Nielsen PF, Huusfeldt PO. Potent derivatives of glucagon-like peptide-1 with pharmacokinetic properties suitable for once daily administration. J Med Chem. 2000;43(9):1664-1669. doi:10.1021/jm9909645

19. Muscogiuri G, Cignarelli A, Giorgino F. GLP-1: benefits beyond pancreas. J Endocrinol Invest. 2014;37(12):1143-1153. doi:10.1007/ s40618-014-0137-y

20. Larsen PJ, Tang-Christensen M, Holst JJ, Ørskov C. Distribution of glucagon-like peptide-1 and other preproglucagon-derived peptides in the rat hypothalamus and brainstem. Neuroscience. 1997;77 (1):257-270. doi:10.1016/s0306-4522(96)00434-4

21. Hunter K, Hölscher C. Drugs developed to treat diabetes, liraglutide and lixisenatide, cross the blood brain barrier and enhance neurogenesis. BMC Neurosci. 2012;13(1):33. doi:10.1186/14712202-13-33

22. Turton M, O'Shea D, Gunn I. A role for glucagon-like peptide-1 in the central regulation of feeding. Nature. 1996;379(6560):69-72. doi: $10.1038 / 379069 \mathrm{a} 0$

23. Holst JJ. The physiology of glucagon-like peptide 1. Physiol Rev. 2007;87(4):1409-1439. doi:10.1152/physrev.00034.2006

24. Heppner KM, Kirigiti M, Secher A. Expression and distribution of glucagon-like peptide-1 receptor mRNA, protein and binding in the male nonhuman primate (macaca mulatta) brain. Endocrinology. 2015;156(1):255-267. doi:10.1210/en.2014-1675

25. Farr OM, Sofopoulos M, Tsoukas MA, et al. GLP-1 receptors exist in the parietal cortex, hypothalamus and medulla of human brains and the GLP-1 analogue liraglutide alters brain activity related to highly desirable food cues in individuals with diabetes: a crossover, randomised, placebo-controlled trial. Diabetologia. 2016;59(5):954-965. doi:10.1007/s00125-016-3874-y

26. Ørskov C, Poulsen SS, Mørten M, Holst JJ. Glucagon-like peptide I receptors in the subfornical organ and the area postrema are accessible to circulating glucagon-like peptide I. Diabetes. 1996;45 (6):832-835. doi:10.2337/diab.45.6.832

27. Flint A, Raben A, Astrup A, Holst JJ. Glucagon-like peptide 1 promotes satiety and suppresses energy intake in humans. $J$ Clin Invest. 1998;101(3):515-520. doi:10.1172/JCI990

28. Näslund E, Gutniak M, Skogar S, Rössner S, Hellström PM. Glucagon-like peptide 1 increases the period of postprandial satiety and slows gastric emptying in obese men. Am J Clin Nutr. 1998;68 (3):525-530. doi:10.1093/ajcn/68.3.525

29. Shughrue PJ, Lane MV, Merchenthaler I. Glucagon-like peptide-1 receptor (GLP1-R) mRNA in the rat hypothalamus. Endocrinology. 1996;137(11):5159-5162. doi:10.1210/endo.137.11.8895391

30. Secher A, Jelsing J, Baquero AF, et al. The arcuate nucleus mediates GLP-1 receptor agonist liraglutide-dependent weight loss. J Clin Invest. 2014;124(10):4473-4488. doi:10.1172/ jci75276

31. Dickson SL, Shirazi RH, Hansson C, Bergquist F, Nissbrandt H, Skibicka KP. The glucagon-like peptide 1 (GLP-1) analogue, exendin-4, decreases the rewarding value of food: a new role for mesolimbic GLP-1 receptors. J Neurosci. 2012;32(14):4812-4820. doi:10.1523/JNEUROSCI.6326-11.2012 
32. Dailey MJ, Moran TH. Glucagon-like peptide 1 and appetite. Trends Endocrinol Metab. 2013;24(2):85-91. doi:10.1016/j. tem.2012.11.008

33. Williams DL, Baskin DG, Schwartz MW. Evidence that intestinal glucagon-like peptide-1 plays a physiological role in satiety Endocrinology. 2009;150(4):1680-1687. doi:10.1210/en.2008-1045

34. Wing RR, Lang W, Wadden TA, et al. Benefits of modest weight loss in improving cardiovascular risk factors in overweight and obese individuals with type 2 diabetes. Diabetes Care. 2011;34 (7):1481-1486. doi:10.2337/dc10-2415

35. Hayes MR, Bradley L, Grill HJ. Endogenous hindbrain glucagon-like peptide-1 receptor activation contributes to the control of food intake by mediating gastric satiation signaling. Endocrinology. 2009;150 (6):2654-2659. doi:10.1210/en.2008-1479

36. Hayes MR, Leichner T, Zhao S, et al. Intracellular signals mediating the food intake-suppressive effects of hindbrain glucagon-like peptide-1 receptor activation. Cell Metab. 2011;13(3):320-330. doi:10.1016/j.cmet.2011.02.001

37. Grossman S. Differentiating incretin therapies based on structure activity, and metabolism: focus on liraglutide. Pharmacotherapy. 2009;29(12P2):25S-32S. doi:10.1592/phco.29.pt2.25S

38. Bjornsdottir I, Olsen A, Larsen U, et al. Metabolism and excretion of the once-daily human GLP-1 analogue liraglutide in healthy subject and its in vitro degradation by dipeptidyl peptidase IV and neutral endopeptidase. Diabetologia. 2008;51:S356-S356.

39. Garber A, Henry R, Ratner R, et al. Liraglutide versus glimepiride monotherapy for type 2 diabetes (LEAD-3 Mono): a randomised, 52-week, Phase III, double-blind, parallel-treatment trial. Lancet. 2009;373(9662):473-481. doi:10.1016/S0140-6736(08)61246-5

40. Yang W, Chen L, Ji Q, et al. Liraglutide provides similar glycaemic control as glimepiride (both in combination with metformin) and reduces body weight and systolic blood pressure in Asian population with type 2 diabetes from China, South Korea and India: a 16-week, randomized, double-blind, active control trial. Diabetes Obes Metab. 2011;13(1):81-88.

41. Ingwersen SH, Khurana M, Madabushi R, et al. Dosing rationale for liraglutide in type 2 diabetes mellitus: a pharmacometric assessment,". J Clin Pharmacol. 2012;52(12):1815-1823. doi:10.1177/ 0091270011430504

42. Ingwersen S, Petri KC, Tandon N, et al. Liraglutide pharmacokinetics and dose-exposure response in Asian subjects with type 2 diabetes from China, India and South Korea. Diabetes Res Clin Pract. 2015;108(1):113-119. doi:10.1016/j.diabres.2015.01.001

43. Damholt B, Golor G, Wierich W, Pedersen P, Ekblom M, Zdravkovic M. An open-label, parallel group study investigating the effects of age and gender on the pharmacokinetics of the once-daily glucagon-like peptide-1 analogue liraglutide. J Clin Pharmacol. 2006;46(6):635-641. doi:10.1177/0091270006288215

44. Petri KCC, Jacobsen LV, Klein DJ. Comparable liraglutide pharmacokinetics in pediatric and adult populations with type 2 diabetes: a population pharmacokinetic analysis. Clin Pharmacokinet. 2015;54 (6):663-670. doi:10.1007/s40262-014-0229-z

45. Buse JB, Rosenstock J, Sesti G, et al. Liraglutide once a day versus exenatide twice a day for type 2 diabetes: a 26-week randomised, parallel-group, multinational, open-label trial (LEAD-6). Lancet. 2009;374(9683):39-47. doi:10.1016/S0140-6736(09)60659-0

46. Flint A, Nazzal K, Jagielski P, Segel S, Zdravkovic M. Influence of hepatic impairment on pharmacokinetics of the long-acting human GLP-1 analogue liraglutide. Diabetes. 2007;56.

47. Buse JB, Garber A, Rosenstock J, et al. Liraglutide treatment is associated with a low frequency and magnitude of antibody formation with no apparent impact on glycemic response or increased frequency of adverse events: results from the liraglutide effect and action in diabetes (LEAD) trials. J Clin Endocrinol Metab. 2011;96 (6):1695-1702. doi:10.1210/jc.2010-2822
48. Scott LJ. Liraglutide: a review of its use in adult patients with type 2 diabetes mellitus. Drugs. 2014;74(18):2161-2174. doi:10.1007/ s40265-014-0321-6

49. Buse JB, Nauck M, Forst T, et al. Exenatide once weekly versus liraglutide once daily in patients with type 2 diabetes (DURATION-6): a randomised, open-label study. Lancet. 2013;381 (9861):117-124. doi:10.1016/S0140-6736(12)61267-7

50. Davies MJ, Bergenstal R, Bode B, et al. Efficacy of liraglutide for weight loss among patients with type 2 diabetes: the SCALE diabetes randomized clinical trial. JAMA. 2015;314(7):687-699. doi:10.1001/ jama.2015.9676

51. Pi-Sunyer X, Astrup A, Fujioka K, et al. A randomized, controlled trial of $3.0 \mathrm{mg}$ of liraglutide in weight management. $N$ Engl J Med. 2015;373(1):11-22. doi:10.1056/NEJMoa1411892

52. Collier A, Blackman A, Foster G, et al. S28 Liraglutide $3.0 \mathrm{Mg}$ Reduces Severity of Obstructive Sleep Apnoea and Body Weight in Obese Individuals with Moderate or Severe Disease: Scale Sleep Apnoea Trial. BMJ Publishing Group Ltd; 2014.

53. Le Roux CW, Astrup A, Fujioka K, et al. 3 years of liraglutide versus placebo for type 2 diabetes risk reduction and weight management in individuals with prediabetes: a randomised, double-blind trial. Lancet. 2017;389(10077):1399-1409. doi:10.1016/S0140-6736(17) 30069-7

54. Knowler WC, Barrett-Connor E, Fowler SE, et al. Reduction in the incidence of type 2 diabetes with lifestyle intervention or metformin. $N$ Engl J Med. 2002;346(6):393-403. doi:10.1056/ nejmoa012512

55. Marso SP, Daniels GH, Brown-Frandsen K, et al. Liraglutide and cardiovascular outcomes in type 2 diabetes. $N$ Engl J Med. 2016;375 (4):311-322. doi:10.1056/NEJMoa1603827

56. Wadden TA, Hollander P, Klein S, et al. Weight maintenance and additional weight loss with liraglutide after low-calorie-diet-induced weight loss: the SCALE maintenance randomized study. Int $J$ Obes. 2013;37(11):1443-1451. doi:10.1038/ijo.2013.120

57. Wadden TA, Tronieri JS, Sugimoto D, et al. Liraglutide $3.0 \mathrm{mg}$ and intensive behavioral therapy (IBT) for obesity in primary care: the SCALE IBT randomized controlled trial. Obesity. 2020;28 (3):529-536. doi:10.1002/oby.22726

58. Drucker DJ, Buse JB, Taylor K, et al. Exenatide once weekly versus twice daily for the treatment of type 2 diabetes: a randomised, open-label, non-inferiority study. Lancet. 2008;372 (9645):1240-1250. doi:10.1016/S0140-6736(08)61206-4

59. Ussher JR, Drucker DJ. Cardiovascular actions of incretin-based therapies. Circ Res. 2014;114(11):1788-1803. doi:10.1161/ CIRCRESAHA.114.301958

60. Nauck M, El-Ouaghlidi A, Hompesch M, Jacobsen J, Elbroend B. No impairment of hypoglycemia counterregulation via glucagon with the long-acting GLP-1 derivative, NN2211, in subjects with Type 2-diabetes. Diabetologia. 2003;46:A285-A285.

61. Marre M, Shaw J, Brändle M, et al. Liraglutide, a once-daily human GLP-1 analogue, added to a sulphonylurea over 26 weeks produces greater improvements in glycaemic and weight control compared with adding rosiglitazone or placebo in subjects with type 2 diabetes (LEAD-1 SU). Diabet Med. 2009;26(3):268-278. doi:10.1111/ j.1464-5491.2009.02666.x

62. Kumaravel A, Zelisko A, Schauer P, Lopez R, Kroh M, Stevens T. Acute pancreatitis in patients after bariatric surgery: incidence, outcomes, and risk factors. Obes Surg. 2014;24(12):2025-2030. doi:10.1007/s11695-014-1337-4

63. Consoli A, Formoso G. Potential side effects to GLP-1 agonists: understanding their safety and tolerability. Expert Opin Drug Saf. 2015;14(2):207-218. doi:10.1517/14740338.2015.987122

64. Bjerre Knudsen L, Madsen LW, Andersen S, et al. Glucagon-like peptide-1 receptor agonists activate rodent thyroid C-cells causing calcitonin release and C-cell proliferation. Endocrinology. 2010;151 (4):1473-1486. doi:10.1210/en.2009-1272 
65. Waser B, Beetschen K, Pellegata NS, Reubi JC. Incretin receptors in non-neoplastic and neoplastic thyroid $\mathrm{C}$ cells in rodents and humans: relevance for incretin-based diabetes therapy. Neuroendocrinology. 2011;94(4):291-301. doi:10.1159/000330447
66. Cotugno M, Nosso G, Saldalamacchia G, et al. Clinical efficacy of bariatric surgery versus liraglutide in patients with type 2 diabetes and severe obesity: a 12-month retrospective evaluation. Acta Diabetol. 2015;52(2):331-336. doi:10.1007/s00592-014-0644-5

\section{Publish your work in this journal}

Clinical Pharmacology: Advances and Applications is an international, peer-reviewed, open access journal publishing original research, reports, reviews and commentaries on all areas of drug experience in humans. The manuscript management system is completely online and

Submit your manuscript here: https://www.dovepress.com/clinical-pharmacology-advances-and-applications-journal includes a very quick and fair peer-review system, which is all easy to use. Visit http://www.dovepress.com/testimonials.php to read real quotes from published authors. 